\title{
Componente del insaponificable del aceite de oliva con polaridad comprendida entre el escualeno y los alcoholes triterpénicos
}

\author{
Por A. Lanzón (1), T. Albi (1), A. Cert (1) y J. Gracián (2) \\ (1) Instituto de la Grasa y sus Derivados. Avda. Padre García Tejero, 4 - 41012 SEVILLA
}

(2) Real Academia Sevillana de Ciencias

\section{RESUMEN}

Componente del insaponificable del aceite de oliva con polaridad comprendida entre el escualeno y los alcoholes triterpénicos

Se ha estudiado y determinado la estructura de un compuesto, de polaridad comprendida entre el escualeno y los alcoholes triterpénicos que se encuentra en el insaponificable de los aceites de oliva refinados y en muchas ocasiones en el de los vírgenes, sobre todo en los de baja calidad.

La espectrometría de masas del mismo y, de sus derivados hidrogenado y sililado, ha puesto de manifiesto que se trata de un alcohol triterpénico lineal, de peso molecular 426 , con el esqueleto similar al del escualeno y con el grupo hidroxilo en posición 2 .

PALABRAS-CLAVE: Aceite de oliva refinado - Alcohol isoprenoide Componente de polaridad intermedia - Insaponificable.

\section{SUMMARY}

Component of the unsaponifiable portion of olive oil with polarity between those of squalene and triterpenic alcohols.

In the unsaponifiable matter of refined olive oils and, frequently, virgin olive oils of bad quality, a new compound with polarity between those of squalene and triterpenic alcohols was isolated.

Mass spectrometry of this compound and of their hydrogenated and sililated derivatives, demonstrated that the compound was a long chain alcohol having 426 of molecular weight, polyisoprenic structure similar to that of squalene, and the hydroxyl group attached at the C-2 carbon atom.

KEY-WORDS: Component of intermediate polarity - Isoprene alcohol - Refined olive oil - Unsaponifiable.

\section{INTRODUCCION}

Al estudiar las alteraciones producidas por los procesos de refinación en el insaponificable del aceite de oliva, nos encontramos, una ligera banda de $\mathrm{Rf}$ algo superior al de la banda de alcoholes lineales y triterpénicos que en algunos casos, según el disolvente de elución utilizado, se solapa con ella. Su análisis por cromatografía de gases en las condiciones normalmente utilizadas para los alcoholes, da lugar a la aparición de dos picos. El primero se encuentra entre el pentacosanol y el hexacosanol, y el segundo entre el último citado y el heptacosanol.

Los estudios realizados en el insaponificable del aceite de oliva para la identificación de compuestos de menor polaridad que los alcoholes, excluidos los hidrocarbu- ros y los tocoferoles, son escasos y, aunque se describen compuestos poli-isoprenoides, le atribuyen estructuras distintas. Así Paquot y Kallel (1973) fraccionaron por cromatografía en capa fina el insaponificable de un aceite de oliva virgen, operando sobre una muestra de aceite enriquecido en insaponificable utilizando una técnica puesta a punto por los mismos autores (Kallel, 1973), consiguiendo asi un aceite con un contenido en insaponificable nueve veces mayor al del aceite original, lo que les permitió poner en evidencia componentes minoritarios difícilmente reconocibles operando a escala usual. Mediante espectrometría de masas, identificaron dos monoalcoholes con un esqueleto hidrocarbonado análogo al del escualeno, suponiendo que el grupo hidroxilo estaba unido a un carbono secundario de la cadena.

Lercker (1985) en el estudio gas cromatográfico de los compuestos de polaridad comprendida entre los alcoholes y los hidrocarburos, de aceites procedentes de aceitunas no maduras, encuentra junto a los tocoferoles unos picos cuyos espectros de masas parecen concordar con una estructura de escualeno epoxidado.

El objetivo del presente trabajo ha sido estudiar la composición de la banda que aparece próxima a la de los alcoholes en CCF que se detecta en casi todos los aceites de oliva vírgenes $y$, con mayor abundancia en los aceites refinados por cualquiera de los dos procedimientos que se utilizan actualmente.

\section{PARTE EXPERIMENTAL}

\subsection{Materiales y aparatos}

Están descritos en su mayoría en un artículo anterior (Lanzón, 1986), el resto de ellos se describen a continuación.

Los espectros de masa se obtuvieron en un espectrómetro AEI, modelo MS-30/VG-70 con el sistema computerizado de datos PDP-11/250, utilizando ionización por impacto electrónico a $70 \mathrm{eV}$, corriente de ionización de $125 \mu \mathrm{A}$ y $200^{\circ} \mathrm{C}$ de temperatura de la fuente.

El espectrómetro estaba acoplado directamente a un cromatógrafo de gases KONIK, modelo KNK-2000C, utili- 
zándose una columna de sílice fundida $(0,25 \mathrm{~mm}$ ø interior $\times 30 \mathrm{~m}$ de longitud) impregnada de fase OV-1 ligada químicamente. Las condiciones cromatográficas fueron: temperatura inicial $80^{\circ} \mathrm{C}$ mantenida durante 2 minutos, programación a $4^{\circ} \mathrm{C} / \mathrm{min}$ hasta $250^{\circ} \mathrm{C}$. Las temperaturas del inyector y de la interfase fueron $250^{\circ} \mathrm{C}$.

\subsection{Metodología analítica}

Se pesan $5 \mathrm{~g}$ de aceite y se saponifican durante 15 minutos con $50 \mathrm{ml}$ de $\mathrm{KOH}$ etanólica $1 \mathrm{~N}$. Una vez concluida la saponificación se añaden $100 \mathrm{ml}$ de agua destilada y se extrae una vez con $100 \mathrm{ml}$ de éter etílico. Se lava posteriormente el extracto etéreo con $40 \mathrm{ml}$ de $\mathrm{KOH}$ acuosa $0,5 \mathrm{~N}$ y luego sucesivamente con porciones de 40 $\mathrm{ml}$ de agua destilada, hasta neutralidad de las aguas de lavado.

El extracto, ya lavado, se trata con sulfato sódico anhidro durante unos 20 minutos agitando ocasionalmente el embudo.

El extracto una vez filtrado se evapora observando que no quede agua, si quedase se añade un poco de acetona y se vuelve a evaporar.

La placa donde se va a cromatografiar el insaponificable se sumerge en una solución etanólica de $\mathrm{KOH}$ $0,5 \mathrm{~N}$ de forma que la cubra hasta unos $3 \mathrm{~cm}$ de su base sacándola al cabo de unos minutos y dejándola secar al aire.

El insaponificable seco se disuelve en $1,5 \mathrm{ml}$ de éter isopropílico y se extienden $0,5 \mathrm{ml}$ de ellos en la placa, desarrollando ésta dos veces con una mezcla de hexano-acetato de etilo 85:15. Finalizados los dos desarrollos se visualiza con yodo, raspándose una banda fina situada por encima de la correspondiente a los alcoholes triterpénicos. Se introduce la sílice raspada en una columnita de elución, realizándose la misma con unos $10 \mathrm{ml}$ de éter isopropílico, concentrándose, posteriormente, para su inyección en el cromatógrafo o para las reacciones de formación de derivados.

\section{Análisis por cromatografía de gases}

Se sigue el método descrito por Lanzón (1990) en el que se utiliza una columna semicapilar de vidrio $(0,75$ $\mathrm{mm} \varnothing$ interno por $25 \mathrm{~m}$ ) impregnada con metil silicona ligada químicamente. Temperatura programada de 110 a $300^{\circ} \mathrm{C}$ con tiempo inicial de 6 minutos y rampa de $5^{\circ} \mathrm{C} /$ min., temperatura del inyector $300^{\circ} \mathrm{C}$ y del detector $320^{\circ} \mathrm{C}$, gas portador $\mathrm{N}_{2}$, con flujo de $14 \mathrm{ml} / \mathrm{min}$. También se ha utilizado una columna capilar de sílice fundida de 0,32 $\mathrm{mm}$ de $\varnothing$ interno por $25 \mathrm{~m}$ impregnada con fenil (5\%)metil silicona ligada químicamente. Temperatura programada de 215 a $280^{\circ} \mathrm{C}$, tiempo inicial 5 minutos con rampa de $0,5^{\circ} \mathrm{C} / \mathrm{min}$, gas portador He con presión en cabeza de $40 \mathrm{KPa}$, relación de división de flujo 1:5, temperatura inyector $275^{\circ} \mathrm{C}$ y del detector $300^{\circ} \mathrm{C}$.

\section{Preparación de derivados hidrogenados y sililados}

La hidrogenación se llevó a cabo con el problema disuelto en $5 \mathrm{ml}$ de hexano, agregándole $0,5-1 \mathrm{mg}$ de catalizador de Adams-Shriner $\left(\mathrm{PtO}_{2}\right)$ y unas gotas de etanol absoluto, haciéndose burbujear en el líquido, a la temperatura ambiente, una corriente de hidrógeno.

La sililación se realizó con el reactivo de sililación constituido por una mezcla 3:1:9 (v:v:v) de hexametildisilasano, trimetilclorosilano y piridina, evaporando previamente la disolución del problema a sequedad y añadiéndole $300 \mu$ l del reactivo.

\section{RESULTADOS Y DISCUSION}

En los registros cromatográficos típicos de la banda separada en cromatografía en capa fina del insaponificable de los aceites de oliva conteniendo los alcoholes grasos y triterpénicos, aparecen dos pequeños picos, señalados como $\mathrm{X}$ y $\mathrm{X}-\mathrm{OH}$ que no se identifican como alcoholes grasos, situándose entre ellos como ya se dijo anteriormente (figura 1).



Figura 1

Cromatograma de gases de la fracción de alcoholes grasos y triterpénicos de un aceite de oliva. Columna vidrio (1/4 pulgada $\times 2 \mathrm{~m}$ ) rellena con OV-101 sobre supelcoport $80-100$. Temperatura inicial $170^{\circ} \mathrm{C}$, 6 min. y luego programada a $4^{\circ} \mathrm{C} / \mathrm{min}$. hasta $245^{\circ} \mathrm{C}$ Portador $\mathrm{N}_{2}$ a $30 \mathrm{ml} / \mathrm{min}$.

Optimizando el desarrollo por cromatografía en capa fina según se indica en la metodología analítica, se aprecia la presencia de una banda débil por encima de la de los alcoholes, integrada fundamentalmente por los componentes a estudiar.

En la fig. 2, se encuentra el registro cromatográfico de la banda antes mencionada, en ella aparecen dos picos, perfectamente definidos, a tiempos de retención de $35,45 \mathrm{~min}$ y $37,11 \mathrm{~min}$, y que identificamos como los mismos que en la fig. 1 se marcaron con $\mathrm{X}$ y $\mathrm{X}-\mathrm{OH}$.

Sobre estos productos se han realizado los estudios orientados a la determinación de sus estructuras.

En primer lugar se obtuvieron sus espectros de masas, el de la fig. 3 es el correspondiente al pico con tiempo de retención de 35,45 min que hemos designado 


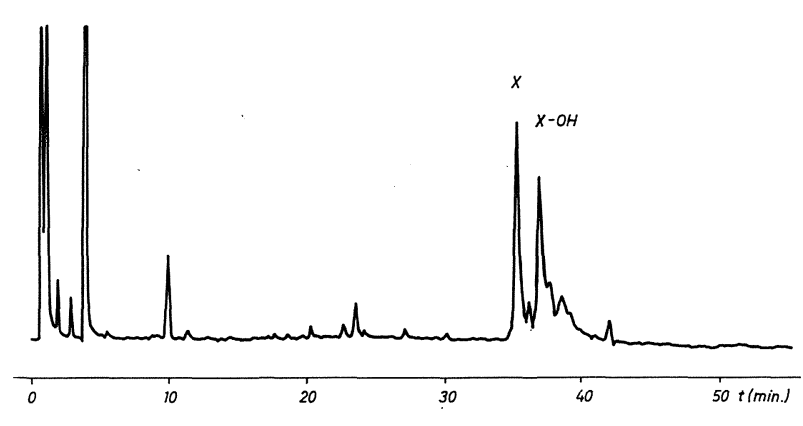

Figura 2

Cromatograma de gases de la fracción de menor polaridad separada de la de los alcoholes grasos y triterpénicos por cromatografía en capa fina. Columna de vidrio $(0,7 \mathrm{~mm} ø$ interno $\times 25 \mathrm{~m})$ impregnada de metil silicona

como X. La estructura de este espectro es muy parecida a la del escualeno pero con un peso molecular de 408 , pudiéndose observar dos series de fragmentos iónicos. La primera de ellas, la constituyen las partículas de $\mathrm{m} / \mathrm{z}$

$m / z 339[\mathrm{M}-69]^{+}\left[\mathrm{M}-\mathrm{CH}_{3}-\mathrm{C}=\mathrm{CH}-\mathrm{CH}_{2}\right]^{+}$
$\mathrm{CH}_{3}$

$\mathrm{m} / \mathrm{z} 271[\mathrm{M}-69-68]^{+}\left[\mathrm{M}-\mathrm{CH}_{3}-\mathrm{C}=\mathrm{CH}-\mathrm{CH}_{2}-\mathrm{CH}_{2}-\mathrm{C}=\mathrm{CH}-\mathrm{CH}_{2}\right]^{+}$

$m / z 203[M-69-2 \times 68]^{+}$

$m / z 135[M-69-3 \times 68]^{+}$

La otra serie está constituida por fragmentos que se registran también en el escualeno, que son los de $\mathrm{m} / \mathrm{z}$ $55,69,81$ y 121 .

Todo ésto pone de manifiesto que el compuesto estudiado parece ser un hidrocarburo lineal de estructura poliisoprenoide, con un peso molecular de 408. La presencia de este hidrocarburo en la zona cromatográfica de los alcoholes, al efectuar la separación en capa fina de la fracción insaponificable del aceite, la explicaremos más adelante discutiéndola en relación al otro componente $\mathrm{X}$ $\mathrm{OH}$, que estudiaremos a continuación.

Al intentar obtener el espectro de masas del producto $\mathrm{X}-\mathrm{OH}$, que es en la fig. 2 el que tiene un tiempo de retención de 37,11 minutos, sólo se conseguía un registro muy pobre y con fuertes variaciones en los resultados, indicando la pequeña cantidad que llegaba a la cámara de ionización del espectrómetro, probablemente por experimentar una fuerte descomposición en el proceso cromatográfico previo. Esta labilidad, unida a posicionarse en el cromatograma del insaponificable en el grupo de los alcoholes, inclinaba a pensar que se trataba de un



Figura 3

Espectro de masas del hidrocarburo lineal de estructura poli-isoprenoide $X$.

alcohol, en cuyo caso podríamos estudiarlo con más seguridad bloqueando el grupo hidroxilo mediante la formación de un silil derivado.

La fracción aislada por cromatografía en capa fina, conteniendo los productos $\mathrm{X}$ y $\mathrm{X}-\mathrm{OH}$ fue sililada, siguiendo la técnica descrita, obteniéndose con el producto resultante el cromatograma de la fig. 4 realizado por inyección en columna capilar para una mejor resolución, en el que el producto $X$, es el separado con un tiempo de retención de 12,31 minutos.

El espectro de masas realizado en este pico es el mismo que el de la fig. 3 al haber permanecido inalterado en la reacción.

El pico situado a 16,82 minutos de retención, es el alcohol sililado X-O-TMS cuyo espectro de masas corresponde a la fig. 5 .

La fragmentación de este compuesto nos induce a pensar que se trata de un alcohol derivado del escualeno, cuyo ión molecular tiene una $\mathrm{m} / \mathrm{z}$ de 498 que correspondería a una fórmula empírica de $\mathrm{C}_{30} \mathrm{H}_{49} \mathrm{O} \mathrm{Si}\left(\mathrm{CH}_{3}\right)_{3}$ y para el que se sugiere la estructura siguiente:



en la que se señalan con líneas verticales discontinuas los lugares de fragmentación de la cadena, siendo previsible los siguientes iones, que se encuentran todos representados en el espectro de la fig. 5 y que para mayor claridad se exponen en la Tabla I.

El pico más abundante es el de $\mathrm{m} / \mathrm{z} 69$ que aparece también en el escualeno, originado por el ión del grupo isopreno terminal, siguiendo una serie de iones originados por la fragmentación de la cadena en posiciones homólogas, dando lugar a una serie de partículas que obedecen a la expresión general. 


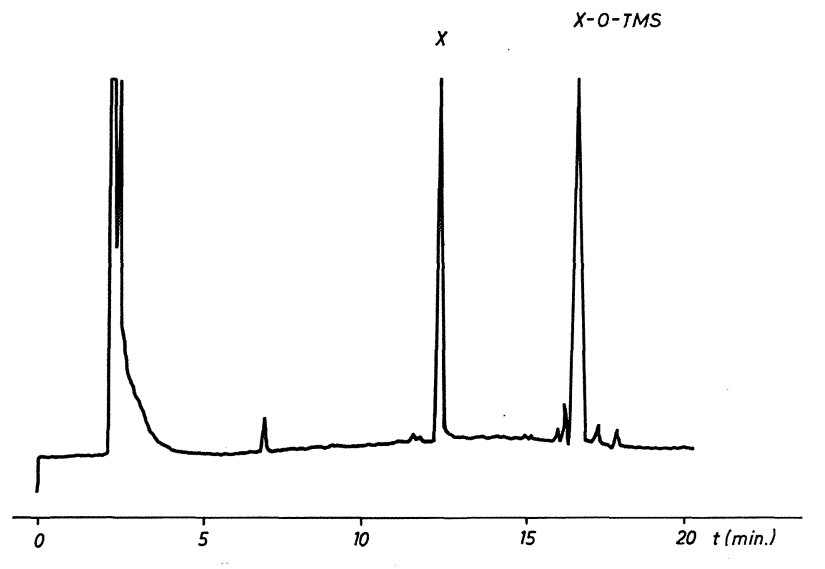

Figura 4

Cromatograma de gases de la fracción de menor polaridad separada de la de los alcoholes grasos y triterpénicos por cromatografía en capa fina. Eteres de trimetilsililo. Columna capilar de silice fundida

$(0,3 \mathrm{~mm} \emptyset$ interno $\times 25 \mathrm{~m})$ impregnada con fenil $(5 \%)$-metil silicona.

$$
\begin{gathered}
{\left[\mathrm{M}-\left(\left(\mathrm{CH}_{3}\right)_{2} \mathrm{C}=\mathrm{CH}-\mathrm{CH}_{2}\right)-\mathrm{n}\left(\mathrm{CH}_{2}-\mathrm{C}=\mathrm{CH}-\mathrm{CH}_{2}\right)\right]^{+}} \\
\mathrm{CH}_{3}
\end{gathered}
$$

Este es un comportamiento típico de una estructura isoprenoide como ya decíamos anteriormente al referirnos al hidrocarburo que correspondía al pico $X$.

$\mathrm{La}$ ausencia de los iones $\mathrm{m} / \mathrm{z} 103,\left(\left(\mathrm{CH}_{3}\right)_{3}-\mathrm{Si}-\stackrel{+}{\mathrm{O}}=\mathrm{CH}_{2}\right)$ y $\mathrm{m} / \mathrm{z} 395,(\mathrm{M}-103)^{+}$, y la aparición de un abundante pico a $\mathrm{m} / \mathrm{z} 131$ están de acuerdo con la presencia de un grupo hidroxilo sililado en la posición 2 de la cadena carbonada.

La eliminación de $\left(\mathrm{CH}_{3}\right)_{3}$-Si-OH da lugar al ión $\mathrm{m} / \mathrm{z}$ 408 del que derivan una serie de iones y a pérdida de grupos isoprénicos en una distribución similar a la encontrada en el espectro del hidrocarburo X. Esta pérdida

Tabla 1

Fragmentación del derivado trimetilsililo del alcohol X-O-TMS

\begin{tabular}{clc}
\hline lón $\mathbf{m} / \mathbf{z}$ & Fragmento & Abundancia relativa (\%) \\
\hline 498 & ión molecular & 15,8 \\
483 & M-CH & 34,1 \\
429 & M-a & 14,6 \\
361 & M-a-b & 4,8 \\
293 & M-a-2b & 2,4 \\
225 & M-a-3b & 12,1 \\
157 & M-a-4b & 24,0 \\
69 & $a$ & 100,0 \\
131 & M & 20,7 \\
408 & M-d-H & 17,0 \\
393 & $408-C H_{3}$ & 6,1 \\
339 & $408-a$ & 24,3 \\
271 & $408-a-b$ & 17,0 \\
203 & $408-a-2 b$ & 3,6 \\
135 & $408-a-3 b$ & 23,1 \\
\hline
\end{tabular}

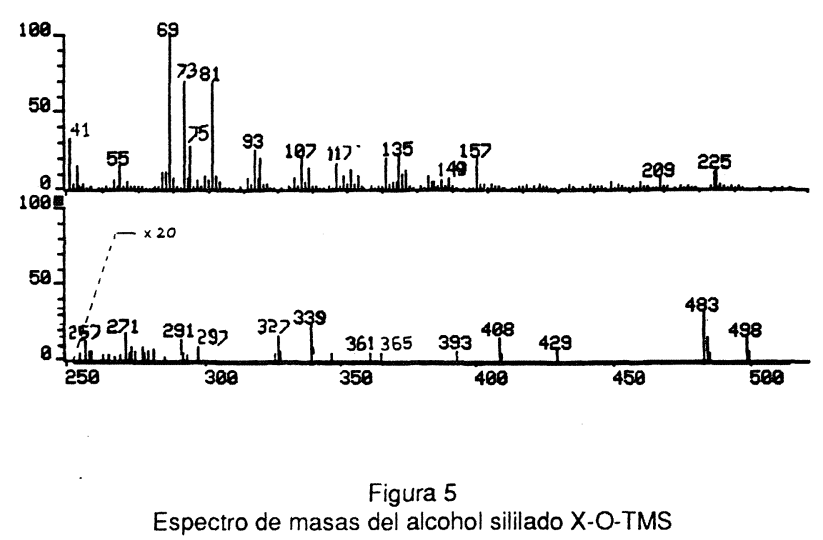

inicial puede ser una reacción iónica o más probablemente una descomposición térmica de la molécula en la fuente de ionización para dar el hidrocarburo $X$.

Los picos a m/z 41, 55, 81, 93 y 107, son los mismos que se encuentran en el espectro del escualeno y que corresponden al extremo izquierdo de la cadena, a través de fragmentaciones y reacciones características de los hidrocarburos poliisoprenoides.

\section{Hidrogenación de la fracción}

La fracción separada por cromatografía en capa fina conteniendo los productos $\mathrm{X}$ y $\mathrm{X}-\mathrm{OH}$, que ya hemos calificado anteriormente como un hidrocarburo y un alcohol de estructuras poli-isoprenoides, análogas a las del escualeno, se hidrogenó, realizándose la cromatografía gaseosa del producto hidrogenado, registrándose un pico pequeño correspondiente a un hidrocarburo $\mathrm{XH}$, un pico muy intenso identificado como el alcohol $\mathrm{XHOH}$ y otros picos menores que, a juzgar por los espectros de masas registrados, deben corresponder a los productos originales parcialmente hidrogenados.

El espectro de masas de XH (fig. 6) acusa la estructura clásica de un hidrocarburo alifático ramificado y monoinsaturado de peso molecular 420. La explicación de la presencia de esta insaturación se dará más adelante al analizar los resultados.

En el espectro de masas del alcohol hidrogenado $\mathrm{XH}$ $\mathrm{OH}$ (fig. 7) aparece un pico mayoritario a $\mathrm{m} / \mathrm{z} 59$, que constituye una clara indicación de tratarse de un alcohol terciario (Seibl, 1973), debido al grupo $-\mathrm{C}(\mathrm{OH})\left(\mathrm{CH}_{3}\right)_{2}$ cuya presencia ya hemos deducido en el análisis del espectro del producto sililado XOTMS

El ión molecular de m/z 438 que cabría esperar de la fórmula postulada antes, para el producto hidrogenado, no aparece en el registro; sin embargo, hay que tener en cuenta, como reconoce Mc Lafferty (1973), que la presencia de un grupo hidroxilo en un alcano desciende su potencia de ionización y, la abundancia del ión molecular decrece y, a menudo, no es observable, favoreciendo la presencia del grupo $\mathrm{OH}$, reacciones de eliminación o fragmentaciones de grupos contiguos.

De acuerdo con ésto, los picos a $\mathrm{m} / \mathrm{z} 418,420,421$ y 423 que se registran en el espectro supuesto un peso 




Figura 6

Espectro de masas del hidrocarburo $\mathrm{XH}$ procedente del alcohol hidrogenado $\mathrm{XH}-\mathrm{OH}$.

molecular de 438 calculado para el "escualanol", podrian tener justificación en las reacciones siguientes:

$$
\begin{aligned}
& \mathrm{M}^{+\cdot}-\mathrm{H}_{2} \mathrm{O}-\mathrm{H}_{2}=418 \\
& \mathrm{M}^{+\cdot}-\mathrm{H}_{2} \mathrm{O}=420 \\
& \mathrm{M}^{+\cdot}-\mathrm{CH}_{3}-\mathrm{H}_{2}=421 \\
& \mathrm{M}^{+\cdot}-\mathrm{CH}_{3}=423
\end{aligned}
$$

Los restantes fragmentos son los que cabe esperar de un alcohol con una larga cadena alifática saturada.

Por tanto el espectro de masas obtenido, es coherente con la fórmula deducida anteriormente para el alcohol original

$$
\mathrm{C}_{27} \mathrm{H}_{43}-\underset{\mathrm{CH}_{3}}{\stackrel{\mathrm{CH}}{\mathrm{CH}}}
$$

Para confirmar aún más dicha estructura, la fracción hidrogenada que estudiamos en el párrafo anterior, se sililó, cromatografiando el producto resultante, obteniéndose únicamente un pico correspondiente al compuesto $\mathrm{XH}-\mathrm{O}-\mathrm{TMS}$ cuyo espectro de masas se indica en la fig. 8.

Si comparamos este espectro con el de $\mathrm{XH}-\mathrm{OH}$ (fig. 7), se ve que presenta una serie de fuertes cambios derivados del bloqueo del hidroxilo por el grupo trimetilsi-

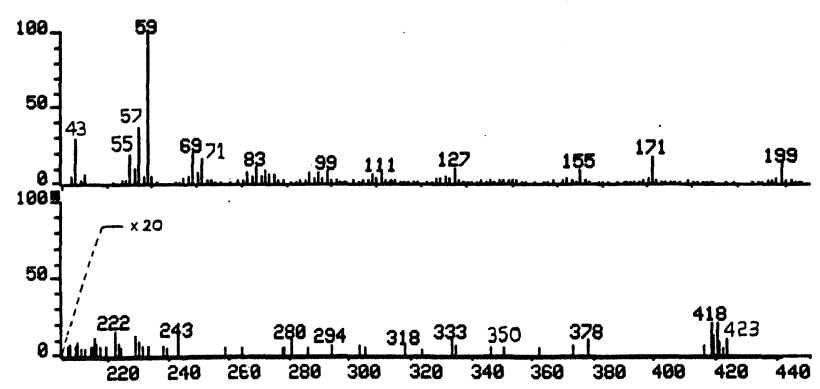

Figura 7

Espectro de masas del alcohol hidrogenado $\mathrm{XH}-\mathrm{OH}$. lilo. El ión molecular de 510, correspondiente al derivado sililado no aparece en el espectro, encontrándose en una gran abundancia iones de $\mathrm{m} / \mathrm{z} 495$, que pueden estar originados por la pérdida de un metilo en la molécula como es normal en los derivados sililados. Aparecen también dos picos a $\mathrm{m} / \mathrm{z} 418$ y 420 , resultante de la pérdida del grupo sililado e hidrógeno, para el primero y el grupo sililado para el segundo, aunque su baja abundancia y la de los fragmentos derivados de estos iones, parece indicar que son consecuencia de reacciones iónicas más que de reacciones térmicas.

$$
\begin{array}{ll}
\mathrm{M}^{+\cdot}-\left(\mathrm{CH}_{3}\right)_{3} \mathrm{SiOH}-\mathrm{H}_{2} & (\mathrm{~m} / \mathrm{z}=418) \\
\mathrm{M}^{+\cdot}-\left(\mathrm{CH}_{3}\right)_{3} \mathrm{SiOH} & (\mathrm{m} / \mathrm{z}=420)
\end{array}
$$

El pico a m/z 59 que en el espectro de la fig. 6 consideramos como característico de un alcohol terciario, en este caso es sustituido por el de $\mathrm{m} / \mathrm{z}$ 131, $\left[\mathrm{C}\left(\mathrm{CH}_{3}\right)_{2}\right.$ $\left.\mathrm{OSi}\left(\mathrm{CH}_{3}\right)_{3}\right]^{+}$que se registra efectivamente con una extraordinaria abundancia (100\%).

Creemos que la causa que motiva la presencia del hidrocarburo $X$ en la fracción de alcoholes, es la deshidratación que sufre el alcohol $\mathrm{XOH}$, por efecto del fuerte calentamiento en el inyector del cromatógrafo gaseoso.

Este proceso está muy favorecido por ser $\mathrm{XOH}$ un alcohol terciario y por la posibilidad de formación de un sistema de dobles enlaces conjugados.

Existen otras observaciones experimentales que son coherentes con el supuesto de que la presencia de hidrocarburos en la fracción de alcoholes, se deba a una deshidratación del alcohol como consecuencia del tratamiento analítico.

En la cromatografía del producto hidrogenado, la presencia de un hidrocarburo monoinsaturado indica que en la fracción de partida no existen hidrocarburos puesto que, de ocurrir este hecho, se obtendría un hidrocarburo totalmente saturado. En consecuencia $\mathrm{XH}$ se forma por deshidratación de $\mathrm{XH}-\mathrm{OH}$ durante la cromatografía. Este extremo se confirma por la ausencia de hidrocarburos en los productos de la reacción de sililación de la fracción hidrogenada, puesto que si existiera algún tipo de hidrocarburo, este debería aparecer.

Por otro lado, el área del pico correspondiente al hidrocarburo monoinsaturado que aparece en el análisis de los productos de hidrogenación, es claramente inferior a la que resulta del cromatograma primitivo. Esto cabe atribuirlo al hecho de una mayor estabilidad del alcohol hidrogenado, ya que no hay posibilidad de conjugación del doble enlace.

En la cromatografía del producto sililado, la relación entre las señales cuantitativas del éter trimetilsililo del alcohol y del hidrocarburo, es sensiblemente mayor, lo cual se justifica al ser la eliminación del TMSOH más difícil que la del agua.

Por último, al sumarse las dos acciones, la de la hidrogenación y bloqueo de la función hidroxilo, el hidrocarburo no aparece ya en el registro cromatográfico, puesto que faltan las dos influencias directrices de la eliminación de agua y TMSOH.

La diferente facilidad con que se producen las reac- 


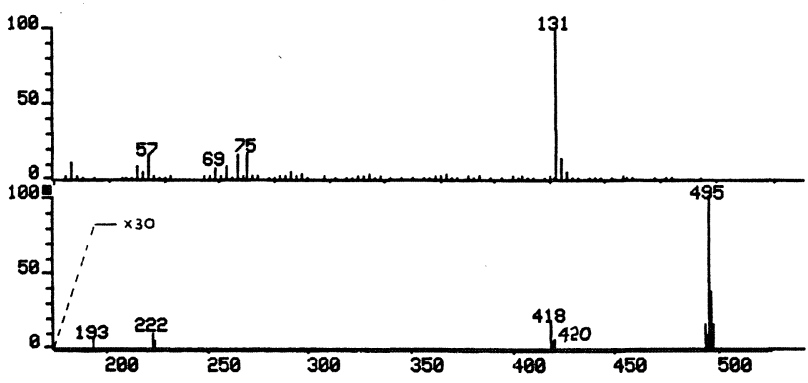

Figura 8

Espectro de masas del alcohol hidrogenado y sililado XH-O-TMS.

ciones de eliminación en los compuestos $\mathrm{X}-\mathrm{OH}, \mathrm{X}$-OTMS y XH-OTMS se ve corroborada por sus espectros de masas que sugieren la existencia de reacciones de descomposición térmica muy importante en el $\mathrm{X}-\mathrm{OH}$ y prácticamente nulas en XH-OTMS.

Como resumen de todo lo dicho, durante la separación por CCF del insaponificable del aceite de oliva suele aparecer una banda de Rf ligeramente superior a la de los alcoholes lineales, que según las condiciones de elución, algunas veces se confunde con ella, y que es debida a un alcohol terciario de estructura poli-isoprenoide que se identifica como 2, 6, 10, 15, 19, 23-hexametiltetracos-3, 6, 10, 14, 18, 22-hexaen-2-ol. Este compuesto es un isómero de otros componentes muy minoritarios identificados por Paquot y Kallel (1973) a los que se atribuye el grupo hidroxilo en un carbono secundario. No se detecta ninguna sustancia a la que pueda atribuirse la estructura de epoxiescualeno.

$\mathrm{El}$ alcohol terciario se descompone térmicamente con gran facilidad para dar el hidrocarburo $\mathrm{C}_{30} \mathrm{H}_{48}$ de peso molecular 408, por lo que aparece como un artefacto en el análisis por cromatografía gaseosa del primero. El espectro de masas de dicho hidrocarburo es similar al de varios componentes de la fracción de hidrocarburos de un aceite de oliva analizado por Bastic y col. (1978). Los análisis de más de 250 fracciones apolares de aceites de oliva realizados por nosotros, han mostrado que estos hidrocarburos aparecen sólo en los aceites refinados, pero en ningún caso en los vírgenes. En consecuencia, postulamos que estos hidrocarburos derivados del escualeno se forman a partir de los diferentes alcoholes isoprénicos por la acción del calor que se aplica a los aceites en los procesos de refinación.

\section{AGRADECIMIENTO}

Este trabajo ha sido financiado por el proyecto ALI880187-CO2-01. Los autores desean expresar su agradecimiento a $\mathrm{D}$. José Luis Ríos Martín por la realización de los espectros de masas.

\section{BIBLIOGRAFIA}

Bastic, M.; Bastic, Lj.; Jovanovic, J.A. and Spiteller, G.- "Hydrocarbons and other weakly polar unsaponifiables in some vegetable oils".- J. Am. Oil Chem. Soc. 55 (1978), 886-891.

Kallel, $H$. et Paquot, C.- "Concentration de la fraction insaponifiable des huiles".- Rev. Fr. Corps Gras 20 (1973), 147-151.

Lanzón, A.; Albi, T. y Gracián, J.- "Influencia del sistema de extracción en algunos componentes del aceite de oliva. Primeros ensayos".- Grasas y Aceites 37 (1986), 254-258.

Lanzón, A.- Tesis Doctoral.- Universidad de Sevilla, 1990.

Lercker, G.- "Recenti acquisizioni analitiche nel settore delle sostanze grasse".- Riv. Ital. Sostanze Grasse 63 (1986), 331-340.

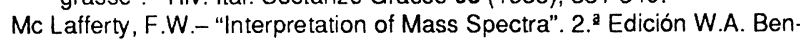
jamin Inc. London (1973) 98

Paquot, C. et Kallel, H.- "Etude de l'insaponifiable de l'huile d'olive et mise en évidence de constituans nouveaux".- Rev. Fr. Corps Gras 20 (1973), 329-333

Seibl, J.- "Espectrometría de masas".- Editorial Alambra. 1. Edición (1973), 125

(Recibido: Diciembre 1991) 